\title{
Tumor growth under rhGM-CSF application in an orthotopic rodent glioma model
}

\author{
THOMAS LINSENMANN ${ }^{1}$, ANNA JAWORK ${ }^{1}$, THOMAS WESTERMAIER ${ }^{1}$, \\ GYÖRGY HOMOLA ${ }^{2}$, CAMELIA MARIA MONORANU ${ }^{3}$, GILES HAMILTON VINCE ${ }^{4}$, \\ ALMUTH FRIEDERIKE KESSLER ${ }^{1}$, RALF-INGO ERNESTUS ${ }^{1}$ and MARIO LÖHR ${ }^{1}$
}

\author{
Departments of ${ }^{1}$ Neurosurgery, ${ }^{2}$ Neuroradiology and ${ }^{3}$ Neuropathology, Julius Maximilians University, \\ Wuerzburg, D-97080 Wuerzburg; ${ }^{4}$ Department of Neurosurgery, Clinical Centre of \\ Aschaffenburg-Alzenau, D-63739 Aschaffenburg, Germany
}

Received September 20, 2018; Accepted February 14, 2019

DOI: $10.3892 / 01.2019 .10179$

\begin{abstract}
Regulation of the host immune response serves a pivotal role in the persistence and progression of malignant glioma. To date, cytotoxic cluster of differentiation $(\mathrm{CD})-8^{+} \mathrm{T}$ and natural killer cells are considered the main cellular components of host tumor control. The influence of macrophages in an orthotropic C6 tumor implantation model was investigated and the aim of the present study was to characterize the effects of systemic macrophage-activation on glioma growth by using the granulocyte macrophage colony stimulating factor (rhGM-CSF). A total of 20 male Sprague-Dawley rats were orthotopically implanted with C6 glioma spheroids and treated subcutaneously with $10 \mu \mathrm{g} / \mathrm{kg}$ rhGM-CSF every other day; 9 animals served as controls. Serial magnetic resonance imaging was performed on days $7,14,21,28,32$ and 42 post-implantation to monitor tumor volume. Histological work-up included hematoxylin and eosin, CD68/ED-1 macrophage, CD8 T-cell and Ki-67 MIB1 proliferation staining in gliomas and spleen. Experimental C6-gliomas developed in 15/20 (75\%) animals. In rhGM-CSF treated rats, tumors developed significantly later and reached a smaller size (median, $134 \mathrm{~mm}^{3}$ ) compared with the controls (median, $262 \mathrm{~mm}^{3}$ ). On day 14, solid tumors presented in 11/17 (65\%) rhGM-CSF-treated animals; in control animals tumor growth was detected in 3/9 animals on day 7 and in all animals on day 14. The mean survival time was 35 days in the rhGM-CSF group and significantly longer when compared with the control group (24 days). Immunohistochemistry exhibited significantly more macrophages in tumors, particularly in the perivascular zone of the rhGM-CSF group when compared with untreated
\end{abstract}

Correspondence to: Dr Thomas Linsenmann, Department of Neurosurgery, Julius Maximilians University Wuerzburg, Josef-Schneider Street 11, D-97080 Wuerzburg, Germany

E-mail: linsenmann_t@ukw.de

Key words: recombinant human granulocyte macrophage colony stimulating factor, glioma, macrophages, microglia, orthotopic rodent glioma model animals; intratumoral $\mathrm{CD} 8^{+}$counts were equal in both groups. A systemic stimulation of macrophages by rhGM-CSF resulted in significantly reduced and delayed tumor growth in the rodent C6 glioma model. The present data suggested a significant role of macrophages in host control of experimental gliomas on the innate immune response. Until now, the role of macrophages may have been underestimated in host glioma control.

\section{Introduction}

Despite advances in surgical techniques, radio- and chemotherapy, the outcome of patients suffering from glioblastoma multiforme (GBM) remains poor, with a median survival of $<15$ months (1). In recent years, immunotherapeutic approaches to malignant glioma have advanced rapidly. This is based on findings revisiting the traditional concept of the central nervous system (CNS) as an immunoprivileged locus. The discovery of the meningeal lymphatic system (2) and an improved understanding of brain $\mathrm{T}$ cell trafficking into the brain via the leptomeninges (3) and the blood-brain-barrier (4) represent important communication channels between the CNS and the peripheral immune system.

Whereas research has focused on T-cells as the critical component of the specific antigen-mediated antitumor response in malignant glioma, there is increasing evidence that non-specific local immunotherapies may aid in glioma defense. Clinical observations that postoperative infections within or close to tumor sites promote a prolonged survival or even complete remission in patients with GBM (5-9) were supported by results of two retrospective single-center studies $(10,11)$ that reported an effect of non-specific systemic immune responses on glioma growth and surveillance. This has been confirmed experimentally with a novel approach using the local administration of heat-inactivated staphylococci as potent immunomodulators in an experimental gliosarcoma model, which led to oncolysis and prolonged survival associated with a distinct peri- and intratumoral infiltration of macrophages (3).

In contrast to the effects of a topical application, it is still debated if systemic immunostimulation exerts a significant antitumor effect. This is the basis and potential key for 
immunotherapies. Non-specific local immunotherapies have proven to be ineffective, whereas specific local immunotherapies have suggested a response (12). Non-specific systemic immunotherapies have not been validated in studies, but numerous suggestions were made regarding effects in allergies and brain abscesses (13). Host factors, including the tumor microenvironment, considerably influence glioma growth and targeting angiogenetic and inflammatory properties recently evolved as an effective treatment strategy (14).

In 2004, spontaneous regression was observed in animal studies with experimental gliomas (15). Spheroids of C6 cells were implanted into the brain and tumor growth was measured by magnetic resonance imaging (MRI)-based volumetry. The aim of the current study was to evaluate effects of a stimulation of the innate immune response, particularly of macrophages, on the proliferation of glioblastoma. For that purpose, recombinant human granulocyte macrophage colony stimulating factor (rhGM-CSF), which has been established in the treatment of neutropenia with a known safety profile, was used in this study.

\section{Materials and methods}

Tumor cell culture and generation of $3 D$ spheroids. Multicellular spheroids from C6 tumor cells (CLS Cell Lines Service $\mathrm{GmbH}$, Eppelheim, Germany) were generated by seeding cells in $75 \mathrm{~cm}^{2}$ culture flasks filled with Dulbecco's modified Eagle's medium (DMEM). Flasks were base-coated with $1 \%$ noble agar (Difco; BD Biosciences, Franklin Lakes, NJ, USA) dissolved in medium. Incubation was performed at $37^{\circ} \mathrm{C}$ in the presence of $5 \% \mathrm{CO}_{2}$ with $100 \%$ humidity. Spheroids were screened for signs of necrosis using inverted light microscopy. Following 6 days, spheroids with a diameter of 200-300 $\mu \mathrm{m}$ without a necrotic core were selected for implantation.

Animal preparation, operative procedure and stimulation of systemic macrophages. The animal care committee of the district authorities (Regierung von Unterfranken, Veterinärwesen, Bavaria, Germany; AZ 55.2-2531.01-65/10) approved the experimental procedures. The implantation procedure has previously been described in detail $(15,16)$. For the current study, 29 male Sprague-Dawley rats (weight, 250-300 g) were anesthetized by an intraperitoneal (i.p.) injection of ketamine hydrochloride (Ketavet ${ }^{\mathrm{TM}}$; Pfizer, Inc., New York, NY, USA) and xylazine hydrochloride (Rompun ${ }^{\mathrm{TM}}$; Bayer AG, Leverkusen, Germany). The animals' heads were fixed in a stereotactic frame using non-perforating bars, a midline incision of the scalp was performed and a $2 \mathrm{~mm}$ burr hole was placed $2 \mathrm{~mm}$ left of the bregma. Following excision of the dura, the cortex was incised in a semicircular fashion using a microscalpel. Under microscopic view, a single spheroid was then placed 2-3 mm subcortically. Following surgery, animals were housed using a 12-h dark/light cycle with free access to food and water and were monitored for signs of discomfort or neurological abnormalities daily. Of the 29 animals implanted with C6 glioma spheroids, 20 rats received a subcutaneous injections of $10 \mu \mathrm{g} / \mathrm{kg}$ rhGSM-CSF every other day. Nine animals served as a control group.

MRI and tumor volumetry. MRI exams were performed on postoperative days (POD) 7, 14, 21, 28, 32 and 42 with a $3 \mathrm{~T}$ clinical scanner (Magnetom Trio ${ }^{\circledR}$; Siemens Healthineers, Erlangen, Germany) using a round surface coil. The following sequences were performed: T1 TSE cor $(0.9 \mathrm{~mm})$, cor T2 TSE rs $(0.7 \mathrm{~mm})$ and cor T2 CISS 3D $(0.3 \mathrm{~mm})$. Animals were anesthetized using $\operatorname{Ketave}^{\circledR}$ as described and were then administered $0.1 \mathrm{ml}$ contrast agent i.p. $10 \mathrm{~min}$ prior to MRI examination. Tumor volumes were calculated using the T2 3D CISS sequences by using MRI Convert ${ }^{\circledR}$ and MIPAV ${ }^{\circledR}$ software.

Tissue preparation. Following each MRI exam, two randomly selected animals were sacrificed for histological examination. Brains and spleens were removed and immediately fixed in paraformaldehyde solution for $24 \mathrm{~h}$ and stored in cold PBS (pH $7.4 ; 4^{\circ} \mathrm{C}$ ) for one week prior to paraffin embedding. For histological studies, spleen sections and coronal brain sections cut into 4- $\mu \mathrm{m}$ slices.

Histological and immunohistochemical analyses. Hematoxylin and eosin (H\&E) staining was performed for an estimation of the gross morphology of tumors and spleen sections.

For immunohistochemistry, sections were stained with Ki67 and CD8 antibodies targeting CD8 ${ }^{+}$lymphocytes (AK CD8 $\alpha$; eBioscience; Thermo Fisher Scientific, Inc., Waltham, MA, USA; cat. no. 14-0084; AK CD68, Zymed; Thermo Fisher Scientific, Inc.; cat. no. 603-2210; AK Ki67; Zymed; Thermo Fisher Scientific, Inc.; cat. no. RMPD 004). Omission of primary antibodies in the control experiments resulted in the expected absence of any cellular labeling. The extent of infiltration of different immune-cell subsets was quantified by cell counting of five representative high-power fields (HPF) in each section, including the tumor margins.

In order to estimate the putative effects of rhGM-CSF stimulation, macrophages and $\mathrm{CD} 8^{+}$lymphocytes in tumor tissues were counted and the corresponding spleen tissue functioned as positive control. Sections were counted at a magnification of x100 using a microscope. In each case, five contiguous fields of view were counted and the mean was determined. For brain sections, five visual fields were counted starting from the tumor margin. As ED1 stains macrophages and microglia, only cells with distinct phagocyte morphology were considered. In $\mathrm{CD}^{+}$cells, only cells with clear lymphocyte morphology were included.

Data analysis. Survival time is presented using box plots. Tumor volumes and cell counts are presented as the median \pm standard deviation. Due of the small sample size, all analyses were of explorative nature. The results (survival times, tumor growth, cell counts) were evaluated graphically. Animals surviving 42 days without MRI-based evidence of tumor growth were excluded from the analysis. Overall survival was assessed by Kaplan-Meier analysis and differences between survival curves were calculated by using Graph Pad Prism ${ }^{\circledR}$ (GraphPad Software, Inc, La Jolla, CA, USA). P<0.05 was considered to indicate a statistically significant difference. Student's t-tests were used in pairwise comparisons.

\section{Results}

MRI studies

rhGM-CSF group. On POD 7, 2/20 (10\%) animals treated with rhGM-CSF developed a visible tumor. Two animals were 


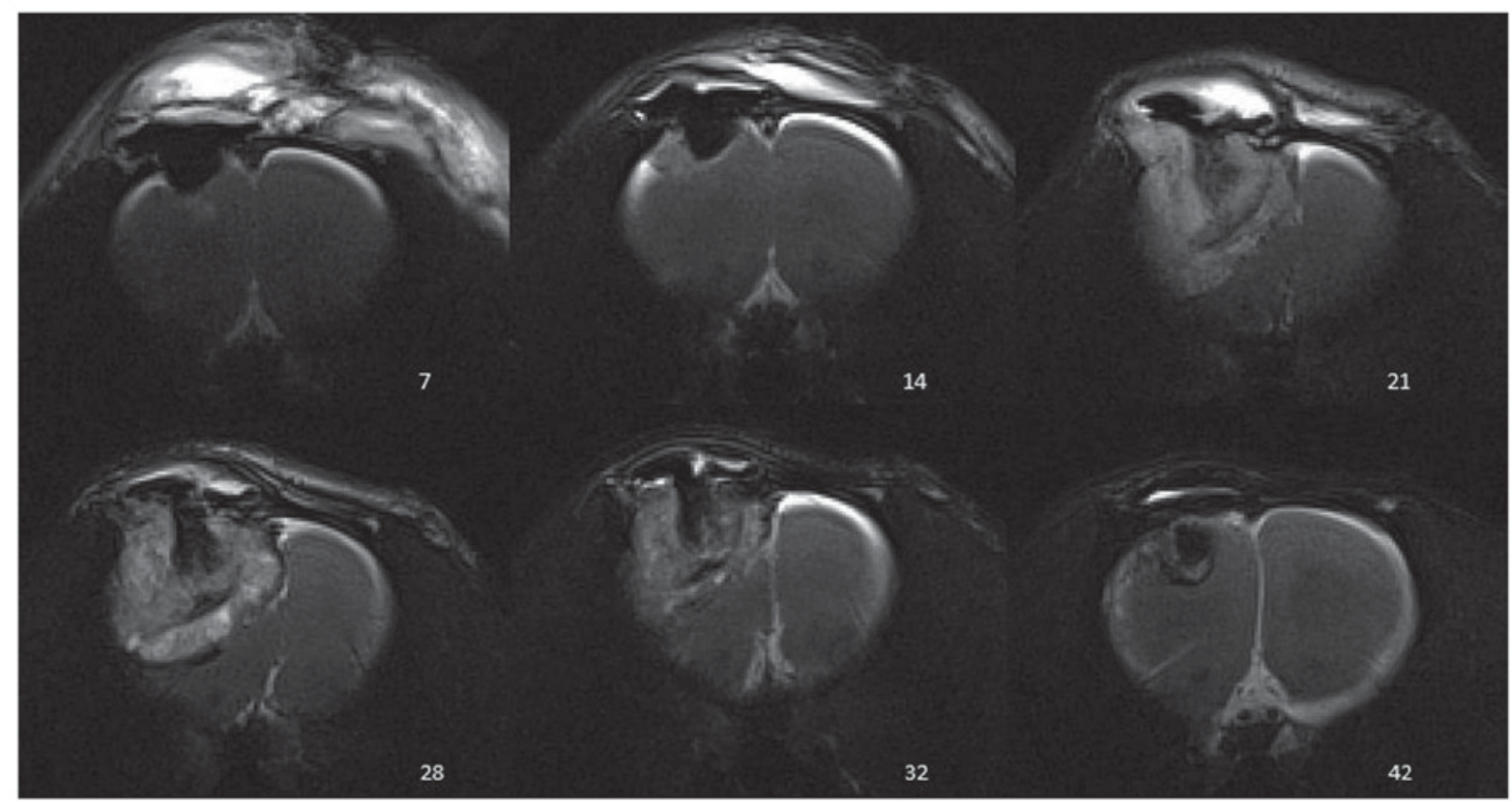

Figure 1. Magnetic resonance imaging performed with a $3 \mathrm{~T}$ clinical scanner. T1 with $0.1 \mathrm{ml}$ contrast medium intraperitoneal: POD 7-42 results are presented. POD 7, extracranial fluid accumulation; POD 14, minimal tumor growth and contrast medium uptake; POD 28, midline shift due to increasing tumor mass accompanied by necrotic changes; POD 32 and 42, decreasing tumor volume, disappearing peritumoral edema and midline shift. Small defects with a remaining fading contrast enhancement. POD, postoperative day.

sacrificed on POD 7 for analysis and further two rats were excluded from the trial due to anesthesia-associated complications, leaving 16 animals for evaluation on POD 14. At that time, solid tumors were observed in 11/16 (65\%) animals and on POD 21, tumors were visible in 14/15 animals. A further animal exhibited a visible tumor on POD 28. Solid tumors developed in 15/20 (75\%) animals. Tumor regression was observed in six (30\%) animals. As a result of severe symptoms associated with tumor growth, seven (35\%) animals of the rhGM-CSF group were sacrificed during the trial.

An exemplary course of tumor growth is presented in Fig. 1. On POD 7, the first extracranial fluid accumulation caused by the surgical trauma was detected. On POD 14, minimal tumor growth with contrast uptake at the edges of the surgical cavity was observed. An increase in size caused peritumoral edema. On POD 28, a midline shift to the opposite side developed due to a mass effect. In addition, the central sparing of contrast enhancement representing necrotic changes was accompanied by a decrease of tumor volume. The peritumoral edema disappeared and midline shift was no longer visible. A small defect with a fading contrast enhancement remained at POD 32 and 42 .

Effects of rhGM-CSF on tumor growth and survival are documented in Figs. 2 and 3. Until POD 20, no significant differences were determined. The mean survival time was 35 days in the rhGM-CSF.

Control group. On POD 7, small tumors were visible in 3/9 (30\%) animals. On POD 14, tumor growth was observed in all control animals. A total of 6/9 (66\%) animals were sacrificed due to severe symptoms of tumor growth. A total of 3 animals were used for histological examination. On POD 28, one animal remained, which was then sacrificed due to tumor mass and occurring symptoms. In contrast to the rhGM-CSF-group, none of the control animals exhibited spontaneous regression

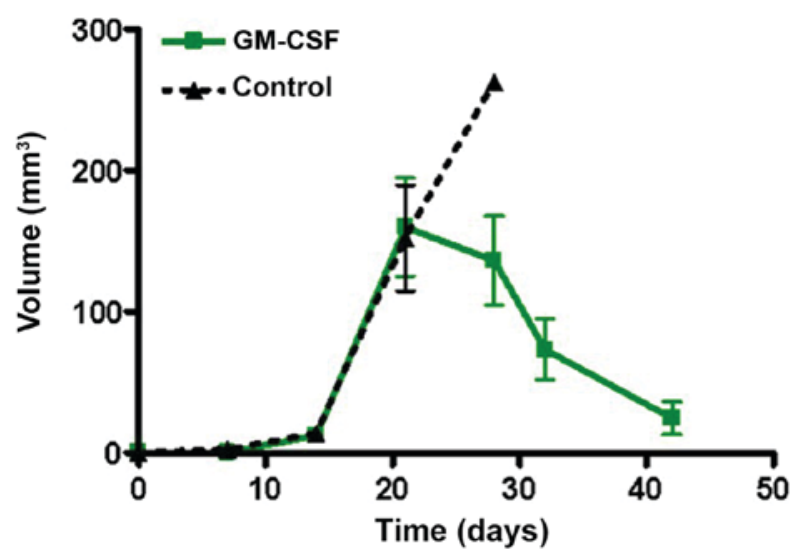

Figure 2. Tumor growth in the rhGM-CSF and control groups. On postoperative day 28 only one measurement of the control group was evaluated and no statistical comparisons were performed. rhGM-CSF, recombinant human granulocyte macrophage colony stimulating factor.

(Fig. 2). On POD 28, only one measurement was obtained for the control group and no statistical comparison was performed.

Comparison of treatment and control groups. Treatment with rhGM-CSF significantly prolonged survival in the rhGM-CSF group compared with the control group (35 vs. 24 days; $\mathrm{P}=0.0343$ ). Tumor volume measurements suggested a delayed growth onset in the rhGM-CSF group compared with the control and a reduced median volume (134 vs. $\left.262 \mathrm{~mm}^{3}\right)$.

Histology. Spheroids were orthotopically implanted and in $15 / 20$ animals tumor growth was observed. In addition, some animals exhibited extracranial tumor components. H\&E staining revealed typical growth characteristics of GBM-type 


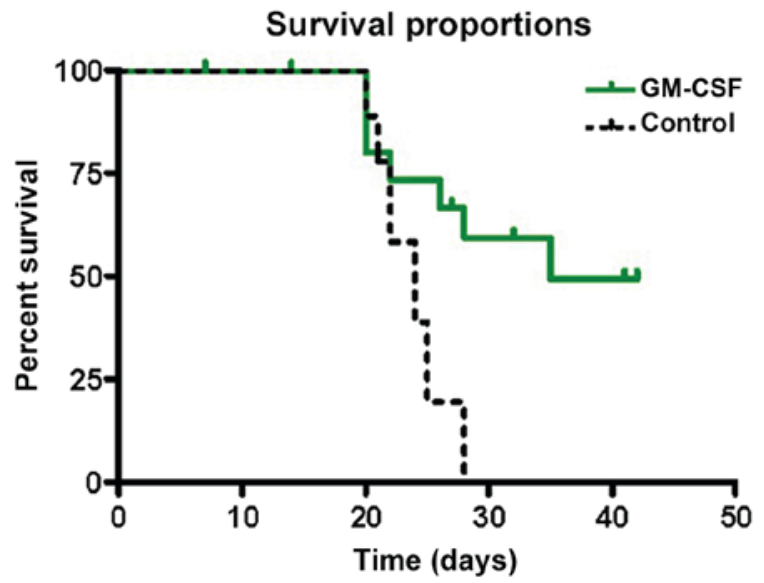

Figure 3. Effects of rhGM-CSF on overall survival. Treatment prolonged survival of animals in the rhGM-CSF group significantly compared with the control (35 vs. 24 days; $\mathrm{P}=0.0343$ ). rhGM-CSF, recombinant human granulocyte macrophage colony stimulating factor.

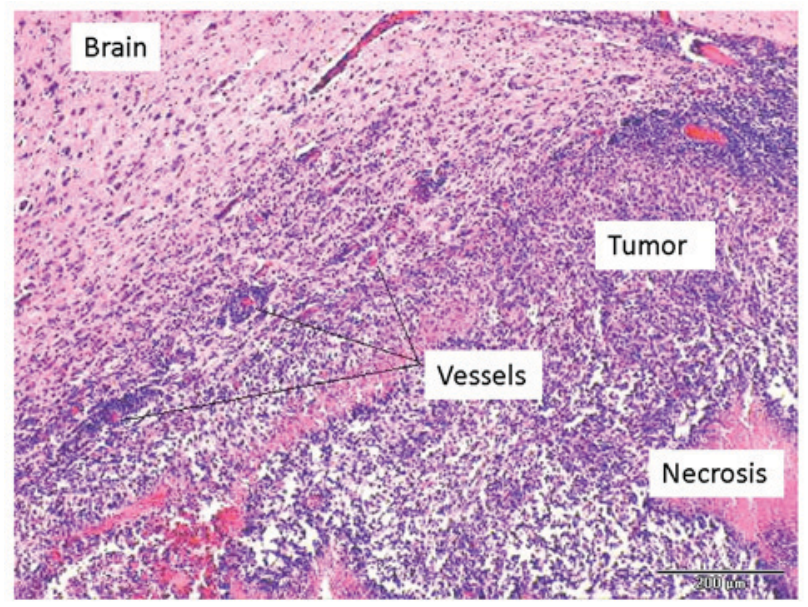

Figure 4. Hematoxylin and eosin staining. Typical growth characteristics of glioblastoma multiforme; neovascularity necrosis and palisade type arrangement of tumor cells are presented. Scale bar, $200 \mu \mathrm{m}$.

neovascularity, necrosis and palisade-type arrangement of the tumor cells (Fig. 4).

$\mathrm{CD} 8^{+}$cells, including cytotoxic T-cells, were monitored to reveal potential effects on tumor growth. There was no significant difference in $\mathrm{CD} 8^{+}$positive cells in the two groups (Fig. 5).

CD68 staining revealed brown-colored macrophages that were located in necrotic areas and in solid tumor tissues (Fig. 6). In addition, numerous macrophages were grouped around tumor vessels (Fig. 7).

Analyzing the time course of macrophage invasion, the maximum number was observed when regression started (Fig. 8). Decreasing tumor size led to decreasing numbers of macrophages. Compared with the control group, rhGM-CSF animals presented a significantly higher numbers of macrophages in brain slices $(\mathrm{P}=0.0275)$.

\section{Discussion}

In the current study, tumor tissues of rhGM-CSF animals exhibited significantly higher numbers of macrophages in

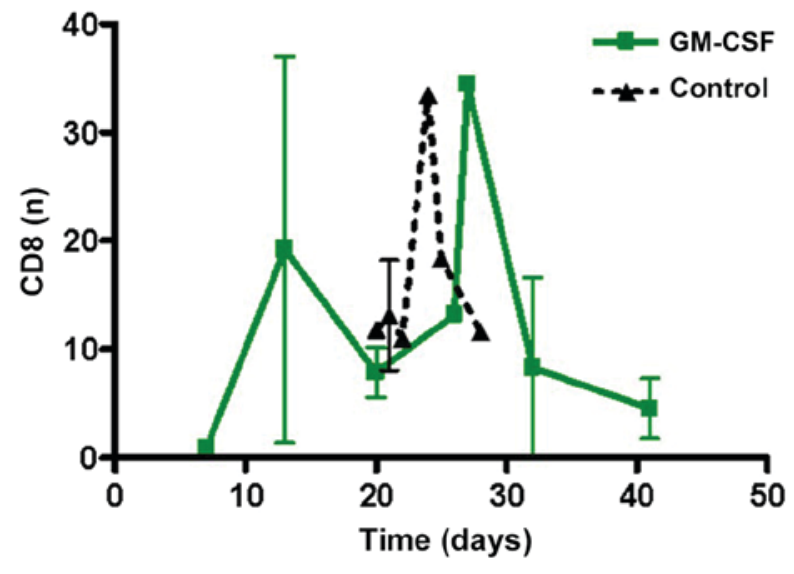

Figure 5. Effects of $\mathrm{CD}^{+}$cells on tumor growth in the rhGM-CSF and control groups. No significant difference in $\mathrm{CD} 8^{+}$cells was observed ( $\mathrm{P}>0.05)$. rhGM-CSF, recombinant human granulocyte macrophage colony stimulating factor. CD, cluster of differentiation; rhGM-CSF, recombinant human granulocyte macrophage colony stimulating factor.

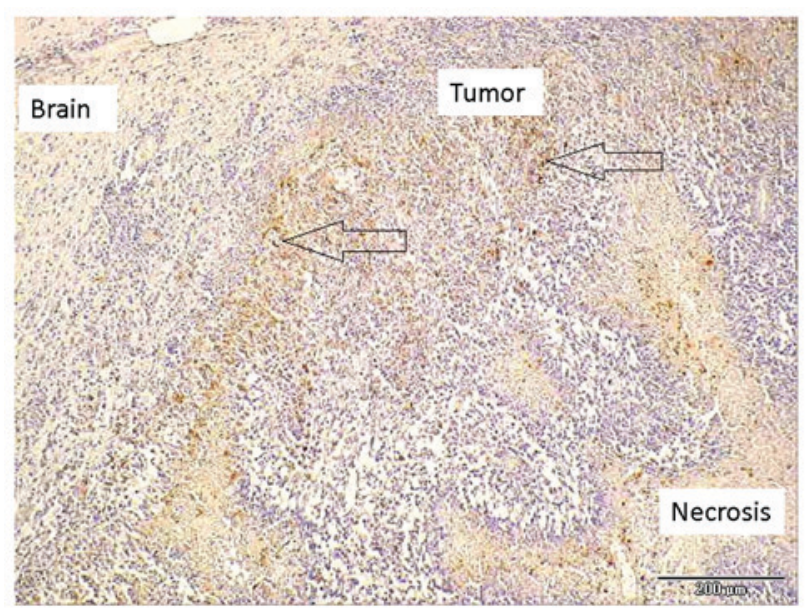

Figure 6. CD68 staining of solid tumor tissues. Macrophages were presented with brown coloring. The arrows indicate macrophages in the solid tumor tissues. Scale bars, $200 \mu \mathrm{m}$. CD, cluster of differentiation.

five-fields-of-view compared with the controls. In addition, animals of the experimental group survived significantly longer compared with animals of the control group. Based on the knowledge that macrophage invasion is correlated with tumor growth in glioblastoma and experimental models, the current data describing reduced tumor growth associated with increased macrophage invasion suggested that the pharmacological induction of macrophages may attenuate or inhibit tumor growth.

A rat brain tumor implantation model was established to investigate macrophage infiltration and tumor growth in rhGM-CSF-treated animals. Compared with mouse models, the larger size of the rat brain facilitates a more precise intracerebral implantation of tumor cells and better in vivo imaging and volumetry of the growing mass (15). Among the various rat brain tumor models, the C6 glioma model has been extensively used and is the best characterized experimental model to investigate a wide array of biological properties of glial tumors, including, their mechanisms of invasion and angiogenesis, intratumoral signaling pathways 


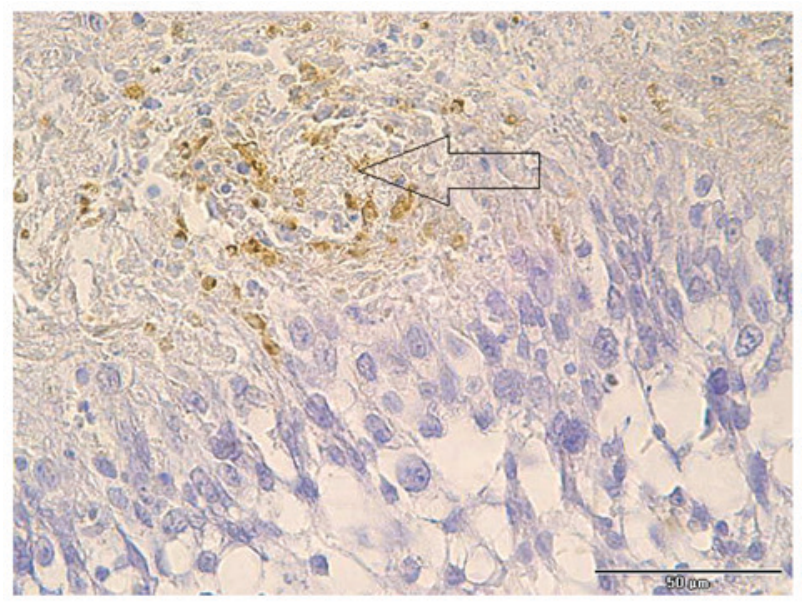

Figure 7. CD68 staining of macrophages around tumor vessels. Macrophages were presented with brown coloring. The arrow indicates macrophages that were grouped around tumor vessels. Scale bar, $50 \mu \mathrm{m}$. CD, cluster of differentiation.

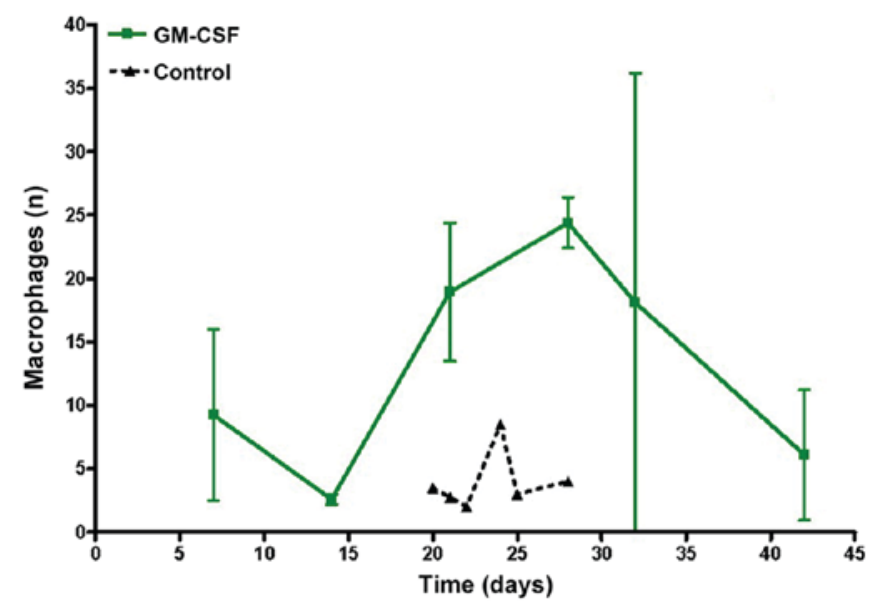

Figure 8. Course of macrophage invasion. A maximum was reached at tumor regression initiation. Decreasing tumor size led to decreasing numbers of macrophages. Compared with the control, rhGM-CSF-treated animals presented a significantly higher number of macrophages in brain slices $(\mathrm{P}=0.0275)$. rhGM-CSF, recombinant human granulocyte macrophage colony stimulating factor.

and efficacy of novel therapeutic modalities $(15,17)$. The C6 glioma model was originally developed by Benda et al (18) by repetitive administration of methylnitrosourea to Wistar rats and was further successfully established in Long-Evans and Sprague-Dawley rats $(19,20)$. C6 cells share certain morphologic features of human glioblastoma-type pleomorphic cells, including nuclear polymorphism, a high mitotic index, areas of necrosis and invasion into the surrounding brain tissue (21). A limitation of the model is its immunogenicity. Several rat strains challenged with C6 cells develop a vigorous immune response. Hence, studies on immunotherapies using the C6 model require careful interpretation (22).

In brain tumors, high numbers of macrophages are detected, increasing with the degree of malignancy $(23,24)$. A total of $5-30 \%$ are typified as microglia/macrophages and account for the majority of infiltrating immune cells in gliomas (25). The majority of studies does not distinguish between microglia and systemic macrophages (CD68 and CD11b/c positive) (26-28). Badie et al (23) have reported that a differentiation of the CD11b/c positive cells is possible through quantification due to low expression of CD45 in microglia and increased expression in macrophages $(1,23,29)$. Initially, it was assumed that high numbers of microglial cells indicate a strong antitumor response in gliomas; however, certain studies postulated a positive effect on tumor growth and a support of the immunosuppressive peritumoral region $(23,30)$. There are various synergistic mechanisms between glioma cells and microglia/macrophages that ensure an immunosuppressant milieu. On the one hand, microglia express low levels of major histocompatibility complex class II (MHC II) in the vicinity of the glioma. The production of transforming growth factor (TGF) $\beta$ by glioma cells causes downregulation of MHC class II expression (31). These molecules are essential in the interaction of antigen-presenting cells and T-lymphocytes (32-34). On the other hand, glioma cells produce substances with immunosuppressive effects. These substances include interleukin (IL) 4, IL 6, TGF $\beta$ [21] and prostaglandin (PG) E2 (32).

Further studies reported a positive effect of high numbers of tumor-macrophages $(35,36)$. Galarneau et al $(37)$ analyzed the influence of macrophages on the growth of glioma cells using a transgenic mouse model. Depletion of the macrophages leads to an increase of tumor volume by $\leq 33 \%$. It is a well-known fact that macrophages are able to recruit $\mathrm{T}$ cells by release of tumor necrosis factor (TNF) (38). However, it remains to be elucidated whether effects on tumor growth are driven by macrophages alone or through TNF-mediated activation of T cells. In addition, a lower vessel density of $\sim 12 \%$ in macrophage-depleted animals was observed. Thus, it is unlikely that increased tumor growth is associated with increased vascular supply (27). Furthermore, Villeneuve et al (38) struggled to determine whether an altered vascular supply in the tumor is caused by the depletion of macrophages.

To determine effects of rhGM-CSF treatment, the M1 and M2 status of macrophages has to be considered. Macrophages present in two different forms, the M1 and M2 status $(32,33,39)$. The M1 status describes classically-activated macrophages associated with inflammation $(22,29)$. In the presence of various cytokines, including rhGM-CSF and interferon $\gamma$, monocytes develop to macrophages with M1 status (33). Expression of signal transducer and activator of transcription (STAT) 1, M1 macrophages exhibit antimicrobial, immunostimulatory and antitumor functions (40). TNF is another factor that contributes to the activation and recruitment of microglia/macrophages. Production of this proinflammatory cytokine contributes to recruitment (33). The M2 status arises from alternative activation and describes macrophages under normal conditions. Among other factors, this status is responsible for preventing excessive immune reactions and explains the more immunosuppressive character of M2 (38). The conversion from M1 to M2 status is induced by IL 4, IL 6, IL 10 and M-CSF. Expression of STAT3 causes M2 macrophage activation, including tissue repair and support of angiogenesis, and favors a tumor progression. This is mediated by the release of various compounds, including IL 10 and TGF $(33,41)$.

During the early stages of glioma development, macrophages are arrested in the M1 tumor suppressive status. The percentage of microglia/macrophages arresting in M2 
correlates with the histological grade of malignancy in glioma $(35,37,39)$. Certain mediators produced by the tumor induce the conversion from M1 to M2; these include IL 4, IL 10, TGF $\beta$ and M-CSF $(35,39,40,42)$. M-CSF produced by tumor cells increases M2 microglia and macrophages and promotes tumor growth and proliferation (40). Reduced tumor growth is detected when inhibiting the M2 status $(42,43)$. Macrophage activation by rhGM-CSF may be a novel therapeutic approach in preserving the M1 status for prolonged periods, preventing a transition into the M2 status (43). Microglial cells treated with rhGM-CSF exhibit upregulated growth and form a heterogeneous cell population in vitro, similar to macrophages. Their function is strongly associated with local environmental factors, such as rhGM-CSF and M-CSF $(30,44)$.

Expression of the Fas-ligand enable macrophage/microgliato support the immunosuppressive environment of gliomas $(41,45)$. There is evidence that cancer stem cells represent the major cause for reprogramming microglia/macrophages to adapt the immunosuppressive M2 status. Tumor stem cells produce soluble (s) CSF, macrophage cytokine 1 (MIC1) and TGF $\beta 1$. These substances polarize microglia/macrophages in the M2 status, block phagocytosis and the production of immunosuppressive cytokines is induced, including IL 10 and TGF $\beta 1$. In addition, inhibition of T-cell proliferation is observed $(41,45,46)$. Macrophages exhibit tumor suppressive functions at later stages in the development of gliomas. Changes may be influenced pharmacologically during this period $(41,47)$. It has been postulated that the therapeutic approach using tumor-associated macrophages is more successful with a staggered induction (43). Considering that different means of activation trigger various responses explains the comparatively late tumor growth observed in a number of rats of the GM-CSF group in the current study. A novel therapeutic approach to treating GBM via targeting macrophages at different states of activity may be considered in the future $(32,39,40)$. Additionally, it has to be clarified, which type of macrophages is promoted by administration of rhGM-CSF that further stimulates tumor progression $(35,48)$. Depletion of rhGM-CSF led to a reduced number of tumor-promoting macrophages and interfered with the development of proinvasive macrophages. In animal studies, rhGM-CSF-depleted animals exhibit increased overall survival (48). In contrast, Grabstein et al (49) described enhanced tumor suppressing properties of macrophages by stimulating with rhGM-CSF in vitro and noted that macrophages exhibit improved antitumor activities.

Effects of rhGM-CSF on an immune system with tumor control are associated with the dose (50). High systemic concentrations lead to recruitment of regulatory $\mathrm{T}$ cells, which contribute to the immunosuppressive environment and interfere with the response against the tumor exerted by the immune system itself. Low doses in the context of vaccinations with tumor antigens lead to an immune stimulation and enhance antitumor activity $(50,51)$. To reduce inter-individual differences, investigations were performed using genetically identical mice. It is postulated that rhGM-CSF at high doses reduces the expression of $\mathrm{M}-\mathrm{CSF}$ receptor, in contrast to a promotion of receptor expression that is observed at low doses $(52,53)$. In humans, treatment with defined amounts of GM-CSF lead to varying serum levels of M-CSF receptor due to individual variance (51).
Data presented in the current study supported the hypothesis of an antitumor effect of rhGM-CSF. The number of macrophages counted in tumor tissues of the rhGM-CSF group was increased compared with the control group. An analysis of macrophage counts and tumor volume over time produced supporting results; initially an increase was observed that towards the end of the trial almost reached baseline levels. It was observed that rhGM-CSF increased macrophage accumulation and exhibited a positive effect on tumor suppression.

In conclusion, a systemic stimulation of macrophages by rhGM-CSF led to significantly reduced and delayed tumor growth in a rodent $\mathrm{C} 6$ glioma model. Model being aware that studies on immunotherapies using the $\mathrm{C} 6$ rat model have to be interpreted carefully because of its immunogenicity.

The host control of experimental gliomas by macrophages may be combined with other promising immune-based approaches, including chimeric antigen receptor $\mathrm{T}$-cell technology or PD-1/PD-L1 checkpoint inhibitors. The role macrophages serve in host tumor control may have been underestimated in the past.

\section{Acknowledgements}

Not applicable.

\section{Funding}

No funding was received.

\section{Availability of data and materials}

The datasets used and/or analyzed during the current study are available from the corresponding author on reasonable request.

\section{Authors' contributions}

TL and AJ performed the experiments to collect the data. TL drafted the manuscript. CMM performed the histological examinations. AFK, TW made substantial contributions to conception and design and analyzing the data. ML made substantial contributions to conception and design, was involved in drafting the manuscript, revised it critically for important intellectual content and gave the final approval of the version to be published. GHV and RIE made substantial contributions to conception and design, interpretation of the data, were involved in drafting the manuscript and revised it critically for important intellectual content. GH performed the neuroradiological examinations. ML was involved in drafting the manuscript and gave final approval for publication. All authors read and approved the final manuscript.

\section{Ethics approval and consent to participate}

The experimental procedures were approved by the Animal Care Committee of the district authorities (Regierung von Unterfranken, Veterinärwesen, Bavaria, Germany; AZ 55.2-2531.01-65/10).

\section{Patient consent for publication}

Not applicable. 


\section{Competing interests}

The authors declare that they have no competing interests.

\section{References}

1. Stupp R, Hegi ME, Mason WP, van den Bent MJ, Taphoorn MJ, Janzer RC, Ludwin SK, Allgeier A, Fisher B, Belanger K, et al: Effects of radiotherapy with concomitant and adjuvant temozolomide versus radiotherapy alone on survival in glioblastoma in a randomised phase III study: 5-year analysis of the EORTC-NCIC trial. Lancet Oncol 10: 459-466, 2009.

2. Louveau A, Smirnov I, Keyes TJ, Eccles JD, Rouhani SJ, Peske JD, Derecki NC, Castle D, Mandell JW, Lee KS, et al: Structural and functional features of central nervous system lymphatic vessels. Nature 523: 337-341, 2015

3. Schläger C, Körner H, Krueger M, Vidoli S, Haberl M, Mielke D, Brylla E, Issekutz T, Cabañas C, Nelson PJ, et al: Effector T-cell trafficking between the leptomeninges and the cerebrospinal fluid. Nature 530: 349-353, 2016.

4. Ratnam NM, Gilbert MR and Giles AJ: Immunotherapy in CNS cancers: The role of immune cell trafficking. Neuro Oncol 21: 37-46, 2019.

5. Bowles AP Jr and Perkins E: Long-term remission of malignant brain tumors after intracranial infection: A report of four cases. Neurosurgery 44: 636-643, 1999.

6. Kapp JP: Microorganisms as antineoplastic agents in CNS tumors. Arch Neurol 40: 637-642, 1983

7. Margolis $\mathbf{J}$ and West $\mathrm{D}$ : Spontaneous regression of malignant disease: Report of three cases. J Am Geriatr Soc 15: 251-253, 1967.

8. Naganuma H, Sasaki A, Satoh E, Nagasaka M, Isoe S, Nakano S and Nukui H: Long-term survival in a young patient with anaplastic glioma. Brain Tumor Pathol 14: 71-74, 1997.

9. Walker DG and Pamphlett R: Prolonged survival and pulmonary metastases after local cure of glioblastoma multiforme. J Clin Neurosci 6: 67-68, 1999.

10. De Bonis P, Albanese A, Lofrese G, de Waure C, Mangiola A Pettorini BL,Pompucci A, Balducci M,Fiorentino A, Lauriola L, et al: Postoperative infection may influence survival in patients with glioblastoma: Simply a myth? Neurosurgery 69: 864-869, 2011.

11. Bohman LE, Gallardo J, Hankinson TC, Waziri AE, Mandigo CE, McKhann GM II, Sisti MB, Canoll P and Bruce JN: The survival impact of postoperative infection in patients with glioblastoma multiforme. Neurosurgery 64: 828-835, 2009.

12. Platten M, Bunse L, Wick W and Bunse T: Concepts in glioma immunotherapy. Cancer Immunol Immunother 65: 1269-1275, 2016

13. Löhr M, Molcanyi M, Poggenborg J, Spuentrup E, Runge M, Röhn G, Härtig W, Hescheler J and Hampl JA: Intracerebral administration of heat-inactivated Staphylococcus epidermidis enhances oncolysis and prolongs survival in a 9L orthotopic gliosarcoma model. Cell Physiol Biochem 31: 614-624, 2013.

14. Thomas AA, Brennan CW, DeAngelis LM and Omuro AM: Emerging therapies for glioblastoma. JAMA Neurol 71: 1437-1444, 2014.

15. Vince GH, Bendszus M, Schweitzer T, Goldbrunner RH, Hildebrandt S, Tilgner J, Klein R, Solymosi L, Christian Tonn J and Roosen K: Spontaneous regression of experimental gliomas-an immunohistochemical and MRI study of the C6 glioma spheroid implantation model. Exp Neurol 190: 478-485, 2004.

16. Goldbrunner RH, Bernstein JJ, Plate KH, Vince GH, Roosen K and Tonn JC: Vascularization of human glioma spheroids implanted into rat cortex is conferred by two distinct mechanisms. J Neurosci Res 55: 486-495, 1999.

17. Grobben B, De Deyn PP and Slegers H: Rat C6 glioma as experimental model system for the study of glioblastoma growth and invasion. Cell Tissue Res 310: 257-270, 2002

18. Benda P, Lightbody J, Sato G, Levine L and Sweet W: Differentiated rat glial cell strain in tissue culture. Science 161: 370-371, 1968

19. Nagano N, Sasaki H, Aoyagi M and Hirakawa K: Invasion of experimental rat brain tumor: Early morphological changes following microinjection of C6 glioma cells. Acta Neuropathol 86 : $117-125,1993$.

20. Whittle IR, MacArthur DC, Malcom GP, Li M, Washington K and Ironside JW: Can experimental models of rodent implantation glioma be improved? A study of pure and mixed glioma cell line tumours. J Neurooncol 36: 231-242, 1998.
21. Chicoine MR and Silbergeld DL: Invading C6 glioma cells maintaining tumorigenicity. J Neurosurg 83: 665-671, 1995.

22. Parsa AT, Chakrabarti I, Hurley PT, Chi JH, Hall JS, Kaiser MG and Bruce JN: Limitations of the C6/Wistar rat intracerebral glioma model: Implications for evaluating immunotherapy. Neurosurgery 47: 993-1000, 2000.

23. Badie B and Schartner J: Role of microglia in glioma biology. Microsc Res Tech 54: 106-113, 2001.

24. Roggendorf W, Strupp S and Paulus W: Distribution and characterization of microglia/macrophages in human brain tumors. Acta Neuropathol 92: 288-293, 1996.

25. Badie B and Schartner JM: Flow cytometric characterization of tumor-associated macrophages in experimental gliomas. Neurosurgery 46: 957-962, 2000

26. da Fonseca AC aand Badie B: Microglia and macrophages in malignant gliomas: Recent discoveries and implications for promising therapies. Clin Dev Immunol 2013: 264124, 2013.

27. Coniglio SJ and Segall JE: Review: Molecular mechanism of microglia stimulated glioblastoma invasion. Matrix Biol 32: 372-380, 2013.

28. Wagner S, Czub S, Greif M, Vince GH, Süss N, Kerkau S, Rieckmann P, Roggendorf $\mathrm{W}$, Roosen $\mathrm{K}$ and Tonn JC: Microglial/macrophage expression of interleukin 10 in human glioblastomas. Int J Cancer 82: 12-16, 1999.

29. Sedgwick JD, Schwender S, Imrich H, Dörries R, Butcher GW and ter Meulen V: Isolation and direct characterization of resident microglial cells from the normal and inflamed central nervous system. Proc Natl Acad Sci USA 88: 7438-7442, 1991.

30. Yang I, Han SJ, Kaur G, Crane C and Parsa AT: The role of microglia in central nervous system immunity and glioma immunology. J Clin Neurosci 17: 6-10, 2010.

31. Czarniecki CW, Chiu HH, Wong GH, McCabe SM and Palladino MA: Transforming growth factor-beta 1 modulates the expression of class II histocompatibility antigens on human cells. J Immunol 40: 4217-4223, 1988.

32. Charles NA, Holland EC, Gilbertson R, Glass R and Kettenmann H: The brain tumor microenvironment. Glia 59: 1169-1180, 2011.

33. Watters JJ, Schartner JM and Badie B: Microglia function in brain tumors. J Neurosci Res 81: 447-455, 2005.

34. Badie B, Bartley B and Schartner J: Differential expression of MHC class II and B7 costimulatory molecules by microglia in rodent gliomas. J Neuroimmunol 133: 39-45, 2002.

35. Hambardzumyan D, Gutmann DH and Kettenmann H: The role of microglia and macrophages in glioma maintenance and progression. Nat Neurosci 19: 20-27, 2016.

36. Zeiner PS, Preusse C, Blank AE, Zachskorn C, Baumgarten P, Caspary L, Braczynski AK, Weissenberger J, Bratzke H, Reiß S, et al: MIF receptor CD74 is restricted to microglia/macrophages, associated with a M1-polarized immune milieu and prolonged patient survival in gliomas. Brain Pathol 25: 491-504, 2015.

37. Galarneau H, Villeneuve J, Gowing G, Julien JP and Vallières L: Increased glioma growth in mice depleted of macrophages. Cancer Res 67: 8874-8881, 2007.

38. Villeneuve J, Tremblay $\mathrm{P}$ and Vallières L: Tumor necrosis factor reduces brain tumor growth by enhancing macrophage recruitment and microcyst formation. Cancer Res 65: 3928-3936, 2005.

39. Komohara Y, Ohnishi K, Kuratsu J and Takeya M: Possible involvement of the M2 anti-inflammatory macrophage phenotype in growth of human gliomas. J Pathol 216: 15-24, 2008.

40. Wei J, Gabrusiewicz K and Heimberger A: The controversial role of microglia in malignant gliomas. Clin Dev Immunol 2013: 285246, 2013.

41. Mantovani A, Marchesi F, Malesci A, Laghi L and Allavena P: Tumour-associated macrophages as treatment targets in oncology. Nat Rev Clin Oncol 14: 399-416, 2017.

42. Alterman RL and Stanley ER: Colony stimulating factor-1 expression in human glioma. Mol Chem Neuropathol 21: 177-188, 1994.

43. Gabrusiewicz K, Ellert-Miklaszewska A, Lipko M, Sielska M, Frankowska M and Kaminska B: Characteristics of the alternative phenotype of microglia/macrophages and its modulation in experimental gliomas. PLoS One 6: e23902, 2011.

44. Fischer HG and Reichmann G: Brain dendritic cells and macrophages/microglia in central nervous system inflammation. J Immunol 166: 2717-2726, 2001.

45. Badie B, Schartner J, Prabakaran S, Paul J and Vorpahl J: Expression of Fas ligand by microglia: Possible role in glioma immune evasion. J Neuroimmunol 120: 19-24, 2001 
46. Wu A, Wei J, Kong LY, Wang Y, Priebe W, Qiao W, Sawaya $\mathrm{R}$ and Heimberger AB: Glioma cancer stem cells induce immunosuppressive macrophages/microglia. Neuro Oncol 12: 1113-1125, 2010

47. Kennedy BC, Maier LM, D'Amico R, Mandigo CE, Fontana EJ, Waziri A, Assanah MC, Canoll P, Anderson RC, Anderson DE and Bruce JN: Dynamics of central and peripheral immunomodulation in a murine glioma model. BMC Immunol 10: 11, 2009.

48. Sielska M, Przanowski P, Wylot B, Gabrusiewicz K, Maleszewska M, Kijewska M, Zawadzka M, Kucharska J, Vinnakota K, Kettenmann H, et al: Distinct roles of CSF family cytokines in macrophage infiltration and activation in glioma progression and injury response. J Pathol 230: 310-321, 2013.

49. Grabstein KH, Urdal DL, Tushinski RJ, Mochizuki DY, Price VL, Cantrell MA, Gillis S and Conlon PJ: Induction of macrophage tumoricidal activity by granulocyte-macrophage colony-stimulating factor. Science 232: 506-508, 1986.

50. Serafini P, Carbley R, Noonan KA, Tan G, Bronte V and Borrello I: High-dose granulocyte-macrophage colony-stimulating factor-producing vaccines impair the immune response through the recruitment of myeloid suppressor cells. Cancer Res 64: 6337-6343, 2004.
51. RapoportAP,AbboudCNandDiPersioJF:Granulocyte-macrophage colony-stimulating factor (GM-CSF) and granulocyte colonystimulating factor (G-CSF): Receptor biology, signal transduction, and neutrophil activation. Blood Rev 6: 43-57, 1992.

52. Gliniak BC and Rohrschneider LR: Expression of the M-CSF receptor is controlled posttranscriptionally by the dominant actions of GM-CSF or multi-CSF. Cell 63: 1073-1083, 1990.

53. McLay RN, Kimura M, Banks WA and Kastin AJ: Granulocyte-macrophage colony-stimulating factor crosses the blood-brain and blood-spinal cord barriers. Brain 120: 2083-2091, 1997.

This work is licensed under a Creative Commons Attribution-NonCommercial-NoDerivatives 4.0 International (CC BY-NC-ND 4.0) License. 